\title{
Two Dimension Threshold Image Segmentation Based on Improved Artificial Fish-Swarm Algorithm
}

\author{
Jiang Suhua \\ School of Basic Science \\ Qingdao Binhai University \\ Qingdao, P.R. China \\ 25524539@qq.com \\ Wang Dongdong \\ School of Basic Science \\ Qingdao Binhai University \\ Qingdao, P.R. China \\ dondonwang@126.com
}

\author{
Liu Chunqiang \\ School of Basic Science \\ Qingdao Binhai University \\ Qingdao, P.R. China \\ 87210661@qq.com
}

\begin{abstract}
To deal with the deficiency of the existing image threshold segmentation algorithm, combining the advantages of Artificial Fish-swarm Algorithm (AFSA), based on the improvement of the AFSA, the thesis proposes a new improved image threshold segmentation algorithm which is the combination of the AFSA and two-dimensional Fisher evaluation function. According to the principle of Fisher pattern recognition, based on two-dimensional histogram, by defining a two-dimensional Fisher criterion functions which is also used as the food concentration function in AFSA, it puts forward a two-dimensional improved image threshold segmentation algorithm. The Experimental results show that the algorithm not only gets the desired segmentation results, but also reduces the time-consuming greatly and thus achieve the fast segmentation goal.
\end{abstract}

Keywords- Artificial Fish-swarm Algorithm; 2-D Fisher criterion;threshold;image segmentation

\section{INTRODUCTION}

Image segmentation is the process of extracting the image into several specific and unique properties according to certain rules, it's the key step of image analysis and object recognition. Image segmentation is the important preprocessing for image recognition and computer vision, so there is no correct recognition without the right segmentation. In the area of communication, image segmentation is very important for the transmission of video phone and other active images, Image processing in the military, remote sensing, meteorology and other large applications is also growing demand.

At present, the main image segmentation methods are threshold segmentation, region segmentation, edge segmentation, histogram, etc.. The threshold segmentation is the most simple and the most common method of segmentation, and the key is the selection of the threshold.
The threshold selection methods have the Ostu method, the gradient statistic method, the maximum entropy method, the histogram Shuangfeng method, etc.. These methods generally exist the disadvantages such as large amount of computation and time, etc..[1,2]

Artificial Fish-swarm Algorithm(AFSA) is a swarm intelligence optimization algorithm based on animal autonomous body, which is proposed by Dr. Li Xiaolei. [3] The main feature of the algorithm is that the special information of the problem is not required to understand, and only need to compare the advantages and disadvantages of the problem. AFSA has a fast convergence rate and strong robustness. In this paper, the behavior of AFSA for finding the 2-D threshold is improved, and applied to image segmentation. In this paper, compared with the exhaustive method, the improved particle swarm algorithm, experiment result illustrates the effectiveness of the algorithm in this paper for finding the optimal 2-D threshold.

\section{TWO-DIMENSIONAL FISHER CRITERION FUNCTION}

The Fisher criterion is a criterion for reducing the dimension in pattern, its purpose is to find an optimal projection vector, the sample of the projection has good separability. Everybody knows, the image threshold divides the image into two kinds of object and background, the more accurate threshold, the separation degree of object and background is better, and the segmentation effect is better. Therefore, the Fisher criterion function is applied to the image segmentation, and the image is divided into two classes of object and background with different gray values. Object and background have the best separation degree when the Fisher criterion function has the maximum value, and the grey value is the best threshold.[2]

Because the traditional one-dimensional histogram 
only reflects the distribution of the gray value of pixels, so when the signal noise ratio of the image is relatively small, the threshold is hard to choose, and the error can be generated. In view of this problem, the 2D Fisher criterion function is introduced to segment the image, and it has strong noise immunity.[4,5]

Suppose the gray level is $\mathrm{L}(\mathrm{L}=0,1,2, \ldots \ldots, 255)$, the gray value of the image at the point $(x, y)$ is represented by function $f(x, y)$.Taking the point $(x, y)$ as the center, the average gray value of the $k \times k$ neighborhood is recorded as function

$$
g(x, y)=\frac{1}{k^{2}} \sum_{m=-k / 2}^{k / 2} \sum_{n=-k / 2}^{k / 2} f(x+m, y+n)
$$

( $1 \leq x \leq w, 1 \leq y \leq h)$, where $w$ and $h$ are the width and height of the image, and usually $k$ is 3.[2]

According to the principle of Fisher criterion, based on the two-dimensional histogram, the 2D Fisher criterion function is defined as follows:

$$
J(s, t)=\frac{\left(\left[\mu_{61}(s), \mu_{62}(t)\right]-\left[\mu_{11}(s), \mu_{2}(t)\right]\right) \times\left(\left[\mu_{61}(s), \mu_{t_{2}}(t)\right]-\left[\mu_{11}(s), \mu_{2}(t)\right]\right)^{T}}{\sigma_{01}^{2}(s)+\sigma_{02}^{2}(t)+\sigma_{11}^{2}(s)+\sigma_{12}^{2}(t)}
$$

Where,

$$
\begin{gathered}
\mu_{01}(s)=\frac{\sum_{i=0}^{s} i \cdot H(i)}{\sum_{i=0}^{s} H(i)}, \mu_{02}(t)=\frac{\sum_{j=0}^{t} j \cdot W(j)}{\sum_{j=0}^{t} W(j)}, \\
\mu_{11}(s)=\frac{\sum_{i=s+1}^{L} i \cdot H(i)}{\sum_{i=s+1}^{L} H(i)}, \mu_{12}(t)=\frac{\sum_{j=t+1}^{L} j \cdot W(j)}{\sum_{j=t+1}^{L} W(j)}, \\
\sigma_{01}^{2}(s)=\sum_{i=0}^{s}\left(i-\mu_{01}(s)\right)^{2} \cdot H(i), \\
\sigma_{02}^{2}(t)=\sum_{j=0}^{t}\left(j-\mu_{02}(t)\right)^{2} \cdot W(j), \\
\sigma_{11}^{2}(s)=\sum_{i=s+1}^{L}\left(i-\mu_{11}(s)\right)^{2} \cdot H(i), \\
\sigma_{12}^{2}(t)=\sum_{j=t+1}^{L}\left(j-\mu_{12}(t)\right)^{2} \cdot W(j), \\
H(i)=\sum_{j=0}^{L-1} N(i, j), i=0,1, \cdots, L, \\
W(j)=\sum_{i=0}^{L-1} N(i, j), j=0,1, \cdots, L .
\end{gathered}
$$

$N(i, j)$ represents the number of pixels when pixel gray value is $f(x, y)=i$ and average gray value of neighborhood pixels is $g(x, y)=j,(i, j=0,1, \cdots, L)$. When $J(s, t)$ takes the maximum value, the corresponding threshold $\left(s^{*}, t^{*}\right)$ is the optimal segmentation threshold.

\section{FINDING TWO-DIMENSION THRESHOLD BASED ON IMPROVED ARTIFICIAL FISH-SWARM ALGORITHM}

\section{A. Correlation definition}

The Artificial Fish(AF)'s position can be denoted by vector $X_{k}=\left(s_{k}, t_{k}\right) \quad\left(0 \leq s_{k}, t_{k} \leq 255\right)$, where $s_{k}, t_{k}(k=1,2, \cdots, N)$ are thresholds which will be searched for optimal values, , and $N$ is the size of the artificial fish swarm. The food consistence of AF's current position is denoted by $F\left(X_{k}\right)=J\left(s_{k}, t_{k}\right)$. The distance of AF $X_{i}$ and $X_{j}$ is denoted by $d_{i j}=\sqrt{\left(s_{i}-s_{j}\right)^{2}+\left(t_{i}-t_{j}\right)^{2}}$. "Visual " denotes the visual distance of AF, “ $\delta$ " denotes the factor of congestion, and “ Step " denotes the maximum step that AF can move for each step. “Try_number" is trying times.

\section{B. The description of AF's behavior}

The main behaviors of artificial fish are random behavior, preying behavior, swarming behavior, and following behavior.

1) The random behavior: Suppose the AF's current position is $X_{i}$, if $X_{i}$ is the optimal position for the present group, its next position is $X_{i \mid \text { next }}=X_{i}$, otherwise, its next position is $X_{i \mid \text { next }}=X_{i}+\operatorname{Random}(-$ Step, Step) . Where, Random(-Step,Step) is a random 2D vector which is composed by random integer in the interval [-Step, Step], the same below.[6]

2) The preying behavior: Suppose the AF's current position is $X_{i}$, and randomly select a position $X_{j}$ in its visual range. If $F\left(X_{j}\right)>F\left(X_{i}\right)$, then $X_{i \mid \text { next }}=X_{j}$, otherwise, randomly select a position in its visual range again, and judge whether the moving condition is satisfied or not. Try Try_number times, if the moving condition is still not satisfied, then the AF carry out the random behavior.

3) The swarming behavior: Suppose the AF's current position is $X_{i}$. Seek the number $n$ of its friends and center position $X_{c}$ in its current neighborhood. If $F\left(X_{c}\right)>F\left(X_{i}\right)$, then it shows that the food consistence is higher. Furthermore, $n / N<\delta$ or the distance $d_{i c}<$ Visual $/ 2$ between $X_{i}$ and $X_{c}$, then $X_{i \mid n \text { ext }}=X_{i}+\operatorname{Random}(0, \operatorname{Step}) \times \operatorname{sign}\left(X_{c}-X_{i}\right)$. Otherwise, carry out the preying behavior.[7,8]

Where, Random(0,Step) is a random 2D vector which is composed by random integer in the interval [0,Step], $\operatorname{sign}\left(X_{c}-X_{i}\right)$ is a $2 \mathrm{D}$ sign vector, that is $\operatorname{sign}\left(X_{c j}-X_{i j}\right)= \begin{cases}-1, & X_{c j}<X_{i j} \\ 0, & X_{c j}=X_{i j}, j=1,2 . \\ 1, & X_{c j}>X_{i j}\end{cases}$

4) The following behavior. Suppose the AF's current position is $X_{i}$. And seek the optimal position $X_{\max }$ of its friends in its current neighborhood. If $F\left(X_{\max }\right)>F\left(X_{i}\right)$, show that position $X_{\max }$ is better than position $X_{i}$. 
Furthermore, $n / N<\delta$ or the distance $d_{i \max }<$ Visual / 2 between $X_{i}$ and $X_{\max }$, then $X_{i \mid n e x t}=X_{i}+\operatorname{Random}(0$, Step $) \times \operatorname{sign}\left(X_{\max }-X_{i}\right)$

Otherwise, carry out the preying behavior.

\section{The bulletin board}

The bulletin board is used to record the optimal position of AF and the food consistence of the optimal position. In the optimizing process, for every AF, we compare the food consistence $F$ of AF's current position with the recording in the bulletin board when AF has been moved once. If the food consistence $F$ of this AF's current position is better than the recording in the bulletin board, then substitute the position of this AF and corresponding $F$ for the previous recording in the bulletin board. In this way, the optimal position of AF has been recorded in the bulletin board.[3]

\section{The end condition of this algorithm}

The end condition of algorithm can be decided according to the properties and the requests of problem. In this algorithm, if the position $X$ in the bulletin board is not changed continuously for $K_{\max }$ times, it shows that the position $X$ is the optimal position, the iteration is terminated, and output the optimal recording of the bulletin board. Otherwise, continue to iterate.

\section{E. The steps of this algorithm}

Step1: Input parameters: Visual , $\delta$, Try_number , $N, K_{\max }$, and randomly generate initial AF swarm in the feasible area, the size of AF swarm is $N$;

Step2: Finding the optimal position of $\mathrm{AF}$ in the current group, record into the bulletin board, $K=0$;

Step3: Each artificial fish simulated swarming behavior and following behavior, choose the best acts as the final executive acts, random behavior and preying behavior are default behaviors;

Step4: Finding the optimal position of AF in the current group, and compare with the position of recording in the bulletin board. If the optimal position of AF in the current group is better than the position of recording in the bulletin board, then replace the position of recording in the bulletin board, $K=0$, otherwise, $K=K+1$;

Step5: If $K=K_{\max }$, then end, and output the position of the bulletin board, otherwise, return Step 3.

\section{EXPERIMENTAL RESULTS}

In order to verify the effectiveness of this algorithm, using this algorithm to segment the Lena image, as shown in Fig.1. Experiments were carried on the Pentium 3.0 $\mathrm{GHz}$ PC using Matlab7.0. [9]The Lena image is changed into the solution of the 2D histogram, as shown in Fig.2. Using exhaustive method, improved particle swarm algorithm[10], and the algorithm in this paper to select threshold and segment image. We get the threshold selection results and image segmentation results as Table I and Fig.3. The parameters of this algorithm in this paper are Visual $=10, \delta=0.618$, Try_number $=5, N=50$, $K_{\max }=20$.

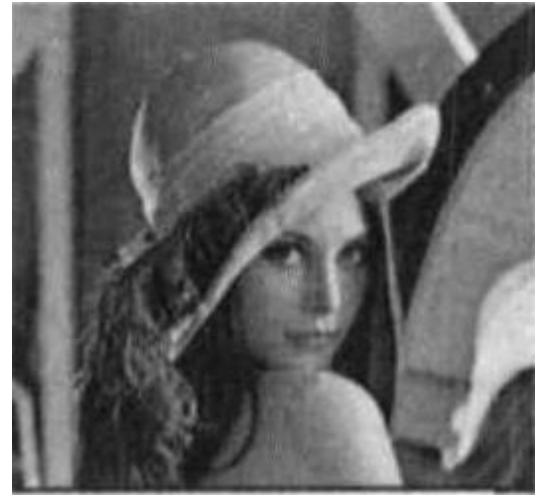

Figure 1. The Lena image.

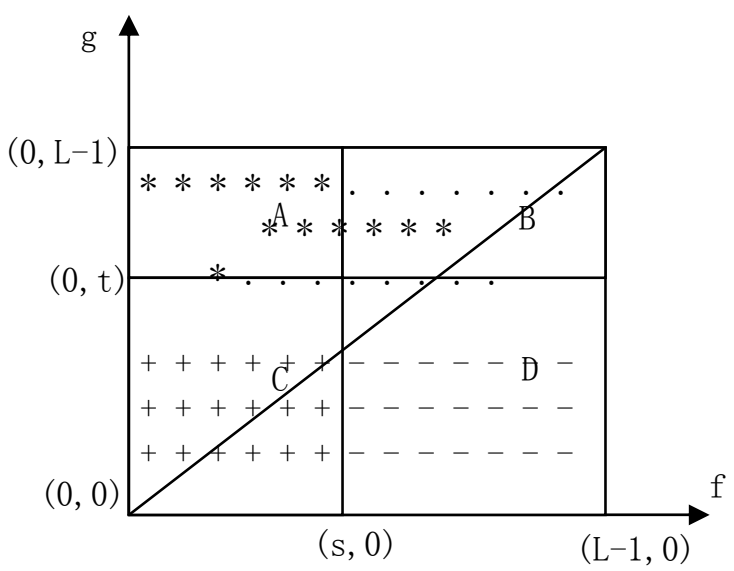

Figure 2. 2D histogram.

TABLE I. RESULTS COMPARISON

\begin{tabular}{|c|c|c|c|c|}
\hline Method & $\begin{array}{c}\text { Optimal } \\
\text { Threshold }\end{array}$ & $\begin{array}{c}\text { Optimal } \\
\text { Target } \\
\text { Value }\end{array}$ & $\begin{array}{c}\text { Running } \\
\text { Time } \\
\text { (s) }\end{array}$ & $\begin{array}{c}\text { Number } \\
\text { of } \\
\text { Success }\end{array}$ \\
\hline $\begin{array}{c}\text { Exhaustive } \\
\text { method }\end{array}$ & $(70,127)$ & 4069.5 & 1806.5420 & - \\
\hline $\begin{array}{c}\text { Improved } \\
\text { particle } \\
\text { swarm } \\
\text { algorithm[10] }\end{array}$ & $(70,127)$ & 4069.5 & 40.7813 & 10 \\
\hline $\begin{array}{c}\text { This } \\
\text { algorithm in } \\
\text { this paper }\end{array}$ & $(70,127)$ & 4069.5 & 39.6021 & 10 \\
\hline
\end{tabular}

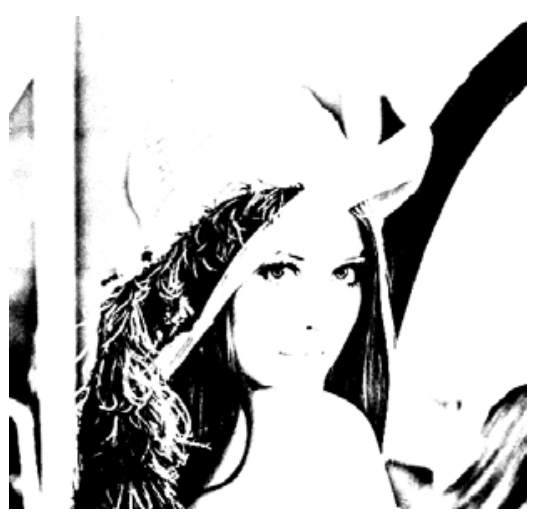

Figure 3. The segmentation result based on this algorithm in this paper. 
The results show that the optimal threshold can be found by this algorithm in this paper, the optimization rate is $100 \%$, and the optimization speed is faster than improved particle swarm algorithm. We have achieved the purpose of the fast segmentation.

\section{CONCLUSIONS}

Image segmentation based on 2D Fisher criterion is an effective method, but compared with one-dimensional, the computation is greatly increased. So, In this paper, we put forward the improved AFSA for finding the optimal 2D threshold vector. The results show that the improved AFSA can effectively search the global optimal 2D threshold vector, and speed of searching is improved. This algorithm is a fast and effective method for image segmentation.

\section{ACKNOWLEDGMENT}

This work was supported by the National Science Foundation of China (61165015) and the National Science Foundation of Qingdao Binhai University (2014K04).

\section{REFERENCES}

[1] Y. Tong, X. H. Qiu,and J. H. Song. “A new algorithm of image segmentation using tow-dimensional histogram based on Fisher criterion function," Telecommunications for Electric Power System,Vol.23, pp.239-244,April 2007.
[2] X. P. Guan, N. Huang,and Y. G. Tang. "Image Segmentation Based on Two-Dimensional Bound Histogram Fisher Criterion and Its Fast Recursive Algorithm,” Information and Control,Vol.38,pp.659-664,September 2009.

[3] X. L. Li, Z. J. Shao, and J. X. Qian. “An optimizing method based on autonomous animats:fish-swarm algorithm,”Theory and Practice of System Engineering,Vol.22,pp.32-38,November 2002.

[4] K. Lu, N. He, and J. Xue. "A new geometric deformable model for medical image segmentation," Chinese Journal of Electronics, Vol.18,pp.200-204,April 2009.

[5] Q. N. Wen,S. R. Wan, and S. Xu "Noise image segmentation using Fisher criterion and regularization level set method,” Journal of Computer Research and Dvelopment, Vol. 49,pp.1339-1347, June 2012.

[6] D. D. Wang, Y. Q. Zhou,and D. Q. Cao. "Artificial Fish-school algorithm for solving nonlinear equations,” Journal of Information and Computational Science,Vol.4, pp.281-289, March 2007.

[7] D. D. Wang. The studying of solving algebraic equation(groups) based on artificial fish school algorithm, Guangxi University for Nationalities,2007.

[8] D. D. Wang, H. J. Pang, Y. J. Chen. "An artificial fish swarm algorithm to determine the real roots of polynomial," Computer engineering \& Science, Vol.34, pp.118-122, September 2012.

[9] D. Mcmahon. Matlab Demystified, Publish Housing of McGraw Hill, 2007.

[10] B. Feng, Z. Wang,and J. Sun."Two dimension threshold image segmentation based on improved particle swarm algorithm," Application Research of Computers,Vol.25,pp.2402-2404, August 2008. 